\title{
Existence of Positive Solutions for Nonlinear Third-Order Boundary Value Problem
}

\author{
Tiaoxia Dun and Pengyu Chen \\ Department of Mathematics, Northwest Normal University, Lanzhou 730070, China \\ Correspondence should be addressed to Pengyu Chen; chpengyu123@163.com
}

Received 13 May 2014; Accepted 8 October 2014; Published 19 October 2014

Academic Editor: Georgios Psihoyios

Copyright (C) 2014 T. Dun and P. Chen. This is an open access article distributed under the Creative Commons Attribution License, which permits unrestricted use, distribution, and reproduction in any medium, provided the original work is properly cited.

We are concerned with the existence of positive solutions for the nonlinear third-order three-point boundary value problem $u^{\prime \prime \prime}(t)+$ $\lambda g(t) f(u(t))=0,0<t<1, u(0)=\alpha u(\eta), u^{\prime}(0)=u^{\prime \prime}(1)=0$, where $0<\eta<1,0<\alpha<1, \lambda$ is a positive parameter, $g:(0,1) \rightarrow[0, \infty)$, and $f:[0, \infty) \rightarrow[0, \infty)$ is continuous. We construct Green's function for the associated linear boundary value problem and obtain some useful properties of Green's function. Finally, by using fixed-point index theorem in cones, we establish the existence results of positive solutions for the boundary value problem an example illustrates the application of the results obtained.

\section{Introduction}

The existence of positive solutions for third-order threepoint boundary value problems has attracted considerable attention by a number of authors. For example, Anderson [1], by using the well-known Guo-Krasnoselskii fixed point theorems [2,3], obtained some existence results for positive solutions for the following boundary value problem (BVP for short):

$$
\begin{gathered}
x^{\prime \prime \prime}(t)=f(t, x(t)), \quad t_{1}<t<t_{3}, \\
x\left(t_{1}\right)=x^{\prime}\left(t_{2}\right), \quad \gamma x\left(t_{3}\right)+\delta x^{\prime \prime}\left(t_{3}\right)=0 .
\end{gathered}
$$

In [4], Sun considered the existence of multiple positive solutions (at least three) to the BVP

$$
\begin{gathered}
u^{\prime \prime \prime}(t)=a(t) f\left(t, u(t), u^{\prime}(t), u^{\prime \prime}(t)\right), \quad 0<t<1, \\
u(0)=\delta u(\eta), \quad u^{\prime}(\eta)=0, \quad u^{\prime \prime}(1)=0,
\end{gathered}
$$

where $\delta \in(0,1), \eta \in[1 / 2,1)$ are constants and $a:(0,1) \rightarrow$ $[0, \infty)$ and $f:[0,1] \times[0, \infty) \times \mathbb{R} \times \mathbb{R} \rightarrow[0, \infty)$ are continuous. The methods used in the paper were based on an application of a fixed point theorem due to Graef and Yang [5].
In [6], the authors considered the existence of positive solution to the third-order three-point BVP

$$
\begin{aligned}
& u^{\prime \prime \prime}(t)+a(t) f(u(t))=0, \quad t \in(0,1), \\
& u(0)=u^{\prime}(0)=0, \quad u^{\prime}(1)=\alpha u^{\prime}(\eta),
\end{aligned}
$$

where $0<\eta<1$ and $1<\alpha<1 / \eta$. The proof relies on GuoKrasnoselskii fixed point theorem.

Motivated by the abovementioned works, in this paper, the fixed-point index theorem in cones was used to discuss the third-order three-point BVP

$$
\begin{gathered}
u^{\prime \prime \prime}(t)+\lambda g(t) f(u(t))=0, \quad 0<t<1, \\
u(0)=\alpha u(\eta), \quad u^{\prime}(0)=u^{\prime \prime}(1)=0,
\end{gathered}
$$

where $0<\eta<1,0<\alpha<1$, and $\lambda$ is a positive parameter. Throughout this paper, we assume that the following conditions are satisfied:

(C1) $f:[0, \infty) \rightarrow[0, \infty)$ is continuous;

(C2) $g \in C([0,1],[0, \infty))$ and is not identically zero on any subinterval of $[0,1]$;

(C3) $0<\int_{0}^{1} s(2-s) g(s) d s<\infty, \int_{\eta}^{1} g(s) d s>0$. 


\section{Preliminaries}

In this section, we will state some important preliminary lemmas.

Let $E=C[0,1], C^{+}[0,1]=\{u \in C[0,1]: u(t) \geq$ $0, t \in[0,1]\}$; then $E$ is a Banach space with norm $\|u\|=$ $\max _{0 \leq t \leq 1}|u(t)|$.

Lemma 1. Assume that $h:(0,1) \rightarrow[0, \infty)$ is continuous, $0<\eta, \alpha<1,0<\int_{0}^{1} s(2-s) h(s) d s<\infty$; then the BVP

$$
\begin{gathered}
u^{\prime \prime \prime}(t)+h(t)=0, \quad 0<t<1, \\
u(0)=\alpha u(\eta), \quad u^{\prime}(0)=u^{\prime \prime}(1)=0
\end{gathered}
$$

has a unique solution

$$
u(t)=\int_{0}^{1} G(t, s) h(s) d s
$$

where

$$
G(t, s)=k(t, s)+\frac{\alpha}{1-\alpha} k(\eta, s)
$$

is corresponding Green's function, and

$$
k(t, s)= \begin{cases}\frac{1}{2} t^{2}-\frac{1}{2}(t-s)^{2}, & 0 \leq s \leq t \leq 1, \\ \frac{1}{2} t^{2}, & 0 \leq t \leq s \leq 1 .\end{cases}
$$

Proof. We can reduce equation $u^{\prime \prime \prime}(t)+h(t)=0$ to an equivalent integral equation

$$
u(t)=-\frac{1}{2} \int_{0}^{t}(t-s)^{2} h(s) d s+a_{2} t^{2}+a_{1} t+a_{0} .
$$

By $u^{\prime}(0)=0$, it follows that $a_{1}=0$; from $u^{\prime \prime}(1)=0$, we get that $a_{2}=(1 / 2) \int_{0}^{1} h(s) d s$, thanks to $u(0)=\alpha u(\eta)$; we have $a_{0}=-(\alpha / 2(1-\alpha)) \int_{0}^{\eta}(\eta-s)^{2} h(s) d s+\left(\alpha \eta^{2} / 2(1-\alpha)\right) \int_{0}^{1} h(s) d s$.

Thus, substituting $a_{0}, a_{2}$ into (9), we have

$$
\begin{aligned}
u(t)= & \frac{1}{2} \int_{0}^{t}\left[t^{2}-(t-s)^{2}\right] h(s) d s+\frac{1}{2} \int_{t}^{1} t^{2} h(s) d s \\
& +\frac{\alpha}{2(1-\alpha)} \int_{\eta}^{1} \eta^{2} h(s) d s \\
& +\frac{\alpha}{2(1-\alpha)} \int_{0}^{\eta}\left[\eta^{2}-(\eta-s)^{2}\right] h(s) d s \\
= & \int_{0}^{1} k(t, s) h(s) d s+\frac{\alpha}{1-\alpha} \int_{0}^{1} k(\eta, s) h(s) d s,
\end{aligned}
$$

where

$$
k(t, s)= \begin{cases}\frac{1}{2} t^{2}-\frac{1}{2}(t-s)^{2}, & 0 \leq s \leq t \leq 1, \\ \frac{1}{2} t^{2}, & 0 \leq t \leq s \leq 1 .\end{cases}
$$

Lemma 2. Let $0<\eta, \alpha<1$, for $G(t, s)$ be given as (7); then we obtain the following results:

(i) $0 \leq G(t, s) \leq((1+\alpha \eta-\alpha) / 2(1-\alpha)) s(2-s), 0 \leq t$, $s \leq 1$,

(ii) $G(t, s) \geq\left(\eta^{2} / 2(1-\alpha)\right) s(2-s), \eta \leq t \leq 1,0 \leq s \leq 1$.

Proof. If $s \leq t$, for all $0 \leq t, s \leq 1$, it follows from (8) that

$$
\begin{aligned}
k(t, s) & =\frac{1}{2} t^{2}-\frac{1}{2}(t-s)^{2}=\frac{1}{2} s(2 t-s) \leq \frac{1}{2} s(2-s) \\
k(t, s) & =\frac{1}{2} t^{2}-\frac{1}{2}(t-s)^{2} \\
& =\frac{1}{2}\left(t^{2} s^{2}-t^{2} s^{2}+2 t^{2} s-2 t^{2} s+2 t s-s^{2}\right) \\
& =\frac{1}{2} t^{2}\left(2 s-s^{2}\right)+\frac{1}{2}\left[2 t s(1-t)-s^{2}\left(1-t^{2}\right)\right] \\
& =\frac{1}{2} t^{2}\left(2 s-s^{2}\right)+\frac{1}{2} s(1-t)(2 t-s-s t) \\
& \geq \frac{1}{2} t^{2}\left(2 s-s^{2}\right)+\frac{1}{2} s(1-t)(s-s t) \\
& =\frac{1}{2} t^{2}\left(2 s-s^{2}\right)+\frac{1}{2} s^{2}(1-t)^{2} \\
& \geq \frac{1}{2} t^{2}\left(2 s-s^{2}\right)
\end{aligned}
$$

and therefore

$$
k(t, s) \geq \frac{1}{2} t^{2} s(2-s)
$$

If $t \leq s$, it follows from (8) that

$$
\begin{aligned}
& k(t, s)=\frac{1}{2} t^{2} \leq \frac{1}{2} s^{2} \leq \frac{1}{2} s(2-s), \\
& k(t, s)=\frac{1}{2} t^{2} \geq \frac{1}{2} t^{2} s(2-s) .
\end{aligned}
$$

By (8), we have

$$
k(\eta, s)= \begin{cases}\frac{1}{2} \eta^{2}-\frac{1}{2}(\eta-s)^{2}, & 0 \leq s \leq \eta \leq 1, \\ \frac{1}{2} \eta^{2}, & 0 \leq \eta \leq s \leq 1,\end{cases}
$$

and thus

$$
\frac{2 k(\eta, s)}{s(2-s)}= \begin{cases}\frac{2 \eta-s}{2-s} \leq \eta, & 0 \leq s \leq \eta \leq 1, \\ \frac{\eta^{2}}{s(2-s)} \leq \frac{\eta^{2}}{s} \leq \eta, & 0 \leq \eta \leq s \leq 1 .\end{cases}
$$

So we obtain that

$$
k(\eta, s) \leq \frac{1}{2} \eta s(2-s)
$$


From (14) and (16), it follows that

$$
k(\eta, s) \geq \frac{1}{2} \eta^{2} s(2-s) .
$$

Hence, by (7), (9), and (16) we get

$$
0 \leq G(t, s) \leq \frac{1+\alpha \eta-\alpha}{2(1-\alpha)} s(2-s), \quad \forall 0 \leq t, s \leq 1,
$$

and by (7), (14), (16), and (20) we have

$$
G(t, s) \geq \frac{\eta^{2}}{2(1-\alpha)} s(2-s), \quad \eta \leq t \leq 1,0 \leq s \leq 1 .
$$

Remark 3. Let $\varphi(s)=(2 \eta-s) /(2-s), 0 \leq s \leq \eta$; then $\varphi^{\prime}(s)=$ $(2 \eta-2) /(2-s)^{2}<0,0 \leq s \leq \eta$, and it follows that

$$
\frac{2 k(\eta, s)}{s(2-s)}=\varphi(s) \leq \varphi(0)=\eta, \quad \text { that is, } k(\eta, s) \leq \frac{1}{2} \eta s(2-s) .
$$

Lemma 4. Assume that $0<\eta, \alpha<1, h:(0,1) \rightarrow[0, \infty)$ is continuous, $0<\int_{0}^{1} s(2-s) h(s) d s<\infty$; then the unique solution $u$ of the BVP (4) is nonnegative and satisfies

$$
\min _{\eta \leq t \leq 1} u(t) \geq \rho\|u\|
$$

where $\rho=\eta^{2} /(1+\alpha \eta-\alpha)$. By $0<\alpha<1<\left(1-\eta^{2}\right) /(1-\eta)$, it is easy to know $0<\rho<1$.

Proof. From (7), (14), (16), and (20), for $t \in[0,1]$, we have

$$
u(t) \geq \frac{1}{2}\left(t^{2}+\frac{\alpha \eta^{2}}{1-\alpha}\right) \int_{0}^{1} s(2-s) h(s) d s \geq 0,
$$

and thus, $u(t)$ is nonnegative.

Let $t \in[0,1]$; by (12) and (19), we get

$$
\begin{aligned}
u(t) & =\int_{0}^{1} k(t, s) h(s) d s+\frac{\alpha}{1-\alpha} \int_{0}^{1} k(\eta, s) h(s) d s \\
& \leq \frac{1}{2} \int_{0}^{1} s(2-s) h(s) d s+\frac{\alpha \eta}{2(1-\alpha)} \int_{0}^{1} s(2-s) h(s) d s \\
& =\frac{1+\alpha \eta-\alpha}{2(1-\alpha)} \int_{0}^{1} s(2-s) h(s) d s,
\end{aligned}
$$

and therefore

$$
\|u\| \leq \frac{1+\alpha \eta-\alpha}{2(1-\alpha)} \int_{0}^{1} s(2-s) h(s) d s .
$$

On the other hand, for $t \in[\eta, 1]$, from Lemma 2 (ii), we have

$$
u(t) \geq \frac{\eta^{2}}{2(1-\alpha)} \int_{0}^{1} s(2-s) h(s) d s \geq \frac{\eta^{2}}{1+\alpha \eta-\alpha}\|u\|,
$$

and thus

$$
\min _{\eta \leq t \leq 1} u(t) \geq \rho\|u\|
$$

where $\rho=\eta^{2} /(1+\alpha \eta-\alpha)$.

Choose a cone $K$ in $E$ as follows:

$$
K=\left\{u \in C^{+}[0,1]: \min _{\eta \leq t \leq 1} u(t) \geq \rho\|u\|\right\} .
$$

Define an operator $A: C^{+}[0,1] \rightarrow C^{+}[0,1]$ by

$$
A u(t)=\lambda \int_{0}^{1} G(t, s) g(s) f(u(s)) d s
$$

By the definition of operator $A$, a positive solution of BVP (4) is equivalent to a nonzero fixed point of $A$.

Lemma 5. Assume that (C1)-(C3) hold; then the operator $A$ : $K \rightarrow K$ is completely continuous.

Proof. By the definition of operator $A$ and Lemma 5, for $u \in$ $K$, it is easy to prove that $A(K) \subset K$. According to the AscoliArzela theorem, we can easily obtain that $A: K \rightarrow K$ is a completely continuous mapping.

Lemma 6 (see [7]). Let $\Omega$ be a bounded open subset of $E$ with $\theta \in \Omega$, and let $A: K \cap \bar{\Omega} \rightarrow K$ be a completely continuous mapping. If $\mu A u \neq u$ for every $u \in K \cap \partial \Omega$ and $0<\mu \leq 1$, then $i(A, K \cap \Omega, K)=1$.

Lemma 7 (see [7]). Let $\Omega$ be a bounded open subset of E, and let $A: K \cap \bar{\Omega} \rightarrow K$ be a completely continuous mapping. If there exists an $v \in K \backslash\{\theta\}$, such that $u-A u \neq \tau v$ for every $u \in K \cap \partial \Omega$ and $\tau \geq 0$, then $i(A, K \cap \Omega, K)=0$.

\section{Main Results}

In this section, we state and prove our main results.

To be convenient, we introduce the following notations:

$$
\begin{gathered}
f^{0}=\limsup _{x \rightarrow 0^{+}} \frac{f(x)}{x}, \quad f_{0}=\liminf _{x \rightarrow 0^{+}} \frac{f(x)}{x}, \\
f^{\infty}=\limsup _{x \rightarrow \infty} \frac{f(x)}{x}, \quad f_{\infty}=\liminf _{x \rightarrow \infty} \frac{f(x)}{x}, \\
B=\left(\max _{0 \leq t \leq 1} \int_{0}^{1} G(t, s) g(s) d s\right)^{-1}, \\
C=\left(\min _{\eta \leq t \leq 1} \int_{\eta}^{1} G(t, s) g(s) d s\right)^{-1} .
\end{gathered}
$$

Our main results are as follows.

Theorem 8. Assume that (C1)-(C3) hold and $f_{\infty}>0, f^{0}<$ $\infty, C / f_{\infty}<B / f^{0}$; then for any $\lambda \in\left(C / f_{\infty}, B / f^{0}\right), B V P(4)$ has at least one positive solution. 
Proof. For every $\lambda \in\left(C / f_{\infty}, B / f^{0}\right)$, according to the condition of $f^{0}<B / \lambda$ and the definition of $f^{0}$, there exist constants $R, \varepsilon_{1}>0$, when $0<u \leq R$; we have $f(u) / u \leq$ $(1 / \lambda)\left(B-\varepsilon_{1}\right)$; namely,

$$
f(u) \leq \frac{1}{\lambda}\left(B-\varepsilon_{1}\right) u \leq \frac{1}{\lambda}\left(B-\varepsilon_{1}\right) R, \quad 0<u \leq R .
$$

Let $\Omega_{1}=\left\{u \in C^{+}[0,1]: \mid\|u\|<R\right\}$, for $u \in K \cap \partial \Omega_{1}$; from (31), we get

$$
\begin{aligned}
\|A u\| & =\max _{0 \leq t \leq 1} \lambda \int_{0}^{1} G(t, s) g(s) f(u(s)) d s \\
& \leq \frac{1}{\lambda}\left(B-\varepsilon_{1}\right) R \max _{0 \leq t \leq 1} \lambda \int_{0}^{1} G(t, s) g(s) d s \\
& =R-\varepsilon_{1} R \max _{0 \leq t \leq 1} \int_{0}^{1} G(t, s) g(s) d s<R .
\end{aligned}
$$

Therefore, for every $u \in K \cap \partial \Omega_{1}, 0<\mu \leq 1$, when $\|u\|=R$, we have $\mu A u \neq u$. In fact, if there exist $u_{0} \in K \cap \partial \Omega_{1}$ and $0<\mu_{0} \leq$ 1 , such that $\mu_{0} A u_{0}=u_{0}$, then $\left\|A u_{0}\right\|=\left(1 / \mu_{0}\right)\left\|u_{0}\right\| \geq\left\|u_{0}\right\|=$ $R$; this is a contradiction. Hence $A$ satisfies the condition of Lemma 6 in $K \cap \partial \Omega_{1}$. By Lemma 6 , we have

$$
i\left(A, K \cap \Omega_{1}, K\right)=1 .
$$

On the other hand, by the condition of $C / f_{\infty}<\lambda$ and the definition of $f_{\infty}$, there exist $H>\rho R, \varepsilon_{2}>0$, and for $u \geq H$, we get $f(u(s)) \geq(1 / \lambda)\left(C+\varepsilon_{2}\right) u$. Let $R_{1}=H / \rho>R, \Omega_{2}=\{u \in$ $\left.C^{+}[0,1]:\|u\|<R_{1}\right\}$; then, $\min \{u(t): t \in[\eta, 1]\} \geq \rho\|u\|=H$, for all $u \in K \cap \partial \Omega_{2}$.

Choose $e(t) \equiv 1, e \in K \backslash\{\theta\}$. We prove that $A$ satisfies the condition of Lemma 7 in $K \cap \partial \Omega_{2}$; namely, $u-A u \neq \tau e$ for every $u \in K \cap \partial \Omega_{2}$ and $\tau \geq 0$. If it is not true, there exist $u_{0} \in K \cap \partial \Omega_{2}$ and $\tau_{0} \geq 0$, such that $u_{0}-A u_{0}=\tau_{0} e$. Let $\sigma=\min \left\{u_{0}(t): t \in[\eta, 1]\right\}, \sigma \geq \rho\left\|u_{0}\right\|=\rho R_{1}$, for $t \in[\eta, 1]$; we have

$$
\begin{aligned}
u_{0}(t) & =\lambda \int_{0}^{1} G(t, s) g(s) f\left(u_{0}(s)\right) d s+\tau_{0} \\
& \geq \frac{1}{\lambda}\left(C+\varepsilon_{2}\right) \sigma \lambda \int_{\eta}^{1} G(t, s) g(s) d s+\tau_{0} \\
& =\sigma+\varepsilon_{2} \sigma \int_{\eta}^{1} G(t, s) g(s) d s+\tau_{0}>\sigma,
\end{aligned}
$$

and this is a contradiction. Thus $A$ satisfies the condition of Lemma 7 in $K \cap \partial \Omega_{2}$. By Lemma 7 we get

$$
i\left(A, K \cap \Omega_{1}, K\right)=0 .
$$

Now, from (35) and (37) it follows that

$$
\begin{aligned}
i\left(A, K \cap\left(\Omega_{2} \backslash \overline{\Omega_{1}}\right), K\right) \\
\quad=i\left(A, K \cap \Omega_{2}, K\right)-i\left(A, K \cap \Omega_{1}, K\right)=-1 .
\end{aligned}
$$

Therefore, $A$ has a fixed point in $K \cap\left(\Omega_{2} \backslash \overline{\Omega_{1}}\right)$, which is a positive solution of BVP (4).
Remark 9. If $f_{\infty}=\infty$, let $C / f_{\infty}=0$; if $f^{0}=0$, let $B / f^{0}=\infty$.

Theorem 10. Assume that (C1)-(C3) hold and $f_{0}>0, f^{\infty}<$ $\infty, C / f_{0}<B / f^{\infty}$; then for any $\lambda \in\left(C / f_{0}, B / f^{\infty}\right), B V P(4)$ has at least one positive solution.

Proof. Let the operator $A$ be defined as (31).

For every $\lambda \in\left(C / f_{0}, B / f^{\infty}\right)$, according to the condition of $f^{\infty}<B / \lambda$ and the definition of $f^{\infty}$, there exist constants $R_{2}, \varepsilon_{3}>0$, and when $u>R_{2}$, we have $f(u) \leq(1 / \lambda)\left(B-\varepsilon_{3}\right) u$. Let $a=\max \left\{f(u): 0 \leq u \leq R_{2}\right\}$; then

$$
f(u) \leq a+\frac{1}{\lambda}\left(B-\varepsilon_{3}\right) u, \quad u \in[0, \infty) .
$$

Choose $R_{3}>a B / \varepsilon_{3} f^{\infty}$; let $\Omega_{3}=\left\{u \in C^{+}[0,1]: \mid\|u\|<R_{3}\right\}$. Then for every $u \in K \cap \partial \Omega_{3}$, we have

$$
\begin{aligned}
\|A u\|= & \max _{0 \leq t \leq 1} \lambda \int_{0}^{1} G(t, s) g(s) f\left(u_{0}(s)\right) d s \\
\leq & \left(a+\frac{1}{\lambda}\left(B-\varepsilon_{3}\right)\|u\|\right) \max _{0 \leq t \leq 1} \lambda \int_{0}^{1} G(t, s) g(s) d s \\
\leq & B\|u\| \max _{0 \leq t \leq 1} \int_{0}^{1} G(t, s) g(s) d s \\
& +\left(a-\frac{1}{\lambda} \varepsilon_{3}\|u\|\right) \lambda \int_{0}^{1} G(t, s) g(s) d s \\
= & R_{3}+\left(a-\frac{1}{\lambda} \varepsilon_{3} R_{3}\right) \frac{\lambda(1+\alpha \eta-\alpha)}{2(1-\alpha)} \int_{0}^{1} s(2-s) g(s) d s \\
< & R_{3} .
\end{aligned}
$$

Thus, for $u \in K \cap \partial \Omega_{3}$ and $0<\mu \leq 1$, when $\|u\|=R_{3}$, we have $\mu A u \neq u$. In fact, if there exist $u_{0} \in K \cap \partial \Omega_{3}$ and $0<\mu_{0} \leq 1$, such that $\mu_{0} A u_{0}=u_{0}$, then $\left\|A u_{0}\right\|=\left(1 / \mu_{0}\right)\left\|u_{0}\right\| \geq\left\|u_{0}\right\|=$ $R_{3}$; this is a contradiction. Hence $A$ satisfies the condition of Lemma 6 in $K \cap \partial \Omega_{3}$. By Lemma 6, we have

$$
i\left(A, K \cap \Omega_{3}, K\right)=1 .
$$

On the other hand, by the condition of $C / f_{0}<\lambda$ and the definition of $f_{0}$, there exist $R_{4}, \varepsilon_{4}>0$, such that $C / \lambda+\varepsilon_{4} \leq f_{0}$, $0<R_{4}<R_{3}$, and when $0<u<R_{4}$, we have $f(u) \geq(C / \lambda+$ $\left.\varepsilon_{4}\right) u$. Let $\Omega_{4}=\left\{u \in C^{+}[0,1]:\|u\|<R_{4}\right\}$. Choose $e(t) \equiv 1$. The process of the proof is similar to Theorem 8 ; we can get $u-A u \neq \tau e$ for every $u \in K \cap \partial \Omega_{4}$ and $\tau \geq 0$. By Lemma 7 we get

$$
i\left(A, K \cap \Omega_{1}, K\right)=0 .
$$

Combining (41), (42), and $\bar{\Omega}_{4} \subset \Omega_{3}$, it follows that

$$
\begin{aligned}
i( & \left.A, K \cap\left(\Omega_{3} \backslash \overline{\Omega_{4}}\right), K\right) \\
\quad & i\left(A, K \cap \Omega_{3}, K\right)-i\left(A, K \cap \Omega_{4}, K\right)=1 .
\end{aligned}
$$

Therefore, $A$ has a fixed point in $K \cap\left(\Omega_{3} \backslash \overline{\Omega_{4}}\right)$, which is a positive solution of BVP (4). 
Remark 11. If $f_{0}=\infty$, let $C / f_{0}=0$; if $f^{\infty}=0$, let $B / f^{0}=0$.

Example 12. We consider the following third-order threepoint BVP:

$$
\begin{aligned}
& u^{\prime \prime \prime}(t)+\lambda \frac{t\left(u+16 u^{3}\right)}{32\left(1+u^{2}\right)}=0, \quad t \in(0,1), \\
& u(0)=\frac{1}{3} u\left(\frac{1}{2}\right), \quad u^{\prime}(0)=u^{\prime \prime}(1)=0
\end{aligned}
$$

where $\lambda$ is a positive parameter; clearly

$$
f(u)=\frac{u+16 u^{3}}{32\left(1+u^{2}\right)}, \quad g(t)=t, \quad \alpha=\frac{1}{3}, \quad \eta=\frac{1}{2} \text {. }
$$

It is easy to obtain that

$$
\begin{gathered}
\int_{0}^{1} s(2-s) g(s) d s=\frac{5}{12}, \quad \int_{1 / 2}^{1} g(s) d s=\frac{3}{8}, \\
f_{\infty}=\frac{1}{2}, \quad f^{0}=\frac{1}{32},
\end{gathered}
$$

and thus, $(C 1)-(C 3)$ hold.

By calculating, we get

$$
B=\frac{288}{77}, \quad C=\frac{384}{27}, \quad \rho=\frac{3}{10} .
$$

By Theorem 8 , for any $C / f_{\infty}=256 / 9<\lambda<B / f^{0}=$ $9216 / 77$, the BVP (44) has at least one positive solution.

\section{Conclusions}

In this work, Green's function for the associated linear boundary value problem was constructed, and some useful properties of the Green's function were obtained. On these bases, the existence results of positive solutions for the boundary value problem were established by using fixed-point index theorem in cones. To demonstrate the applications of our results, an example is given.

\section{Conflict of Interests}

The authors declare that there is no conflict of interests regarding the publication of this paper.

\section{Acknowledgments}

This research is supported by NNSF of China (11261053) and NSF of Gansu province (1208R-JZA129).

\section{References}

[1] D. R. Anderson, “Green's function for a third-order generalized right focal problem," Journal of Mathematical Analysis and Applications, vol. 288, no. 1, pp. 1-14, 2003.
[2] D. Guo and V. Lakshmikantham, Nonlinear Problems in Abstract Cones, Academic Press, New York, NY, USA, 1998.

[3] M. A. Krasnoselskiŭ, Positive Solutions of Operator Equations, P. Noordhoff, Groningen, The Netherlands, 1964.

[4] Y. Sun, "Existence of triple positive solutions for a third-order three-point boundary value problem," Journal of Computational and Applied Mathematics, vol. 221, no. 1, pp. 194-201, 2008.

[5] J. R. Graef and B. Yang, "Positive solutions of a nonlinear third order eigenvalue problem," Dynamic Systems and Applications, vol. 15, no. 1, pp. 97-110, 2006.

[6] L. J. Guo, J. P. Sun, and Y. H. Zhao, "Existence of positive solutions for nonlinear third-order three-point boundary value problems," Nonlinear Analysis. Theory, Methods \& Applications, vol. 68, no. 10, pp. 3151-3158, 2008.

[7] D. Guo and J. Sun, "Calculation and application of topological degree," Journal of Mathematical Research and Exposition, vol. 8, pp. 469-480, 1988 (Chinese). 


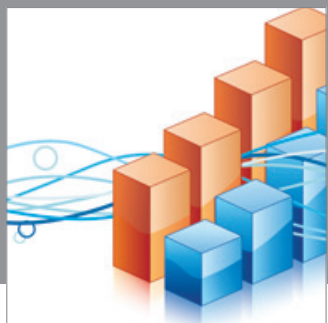

Advances in

Operations Research

mansans

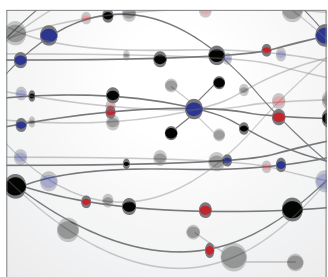

The Scientific World Journal
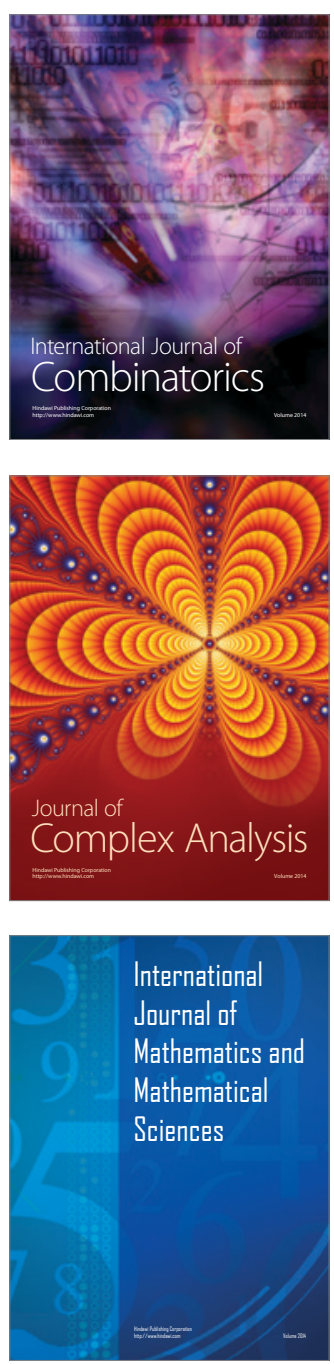
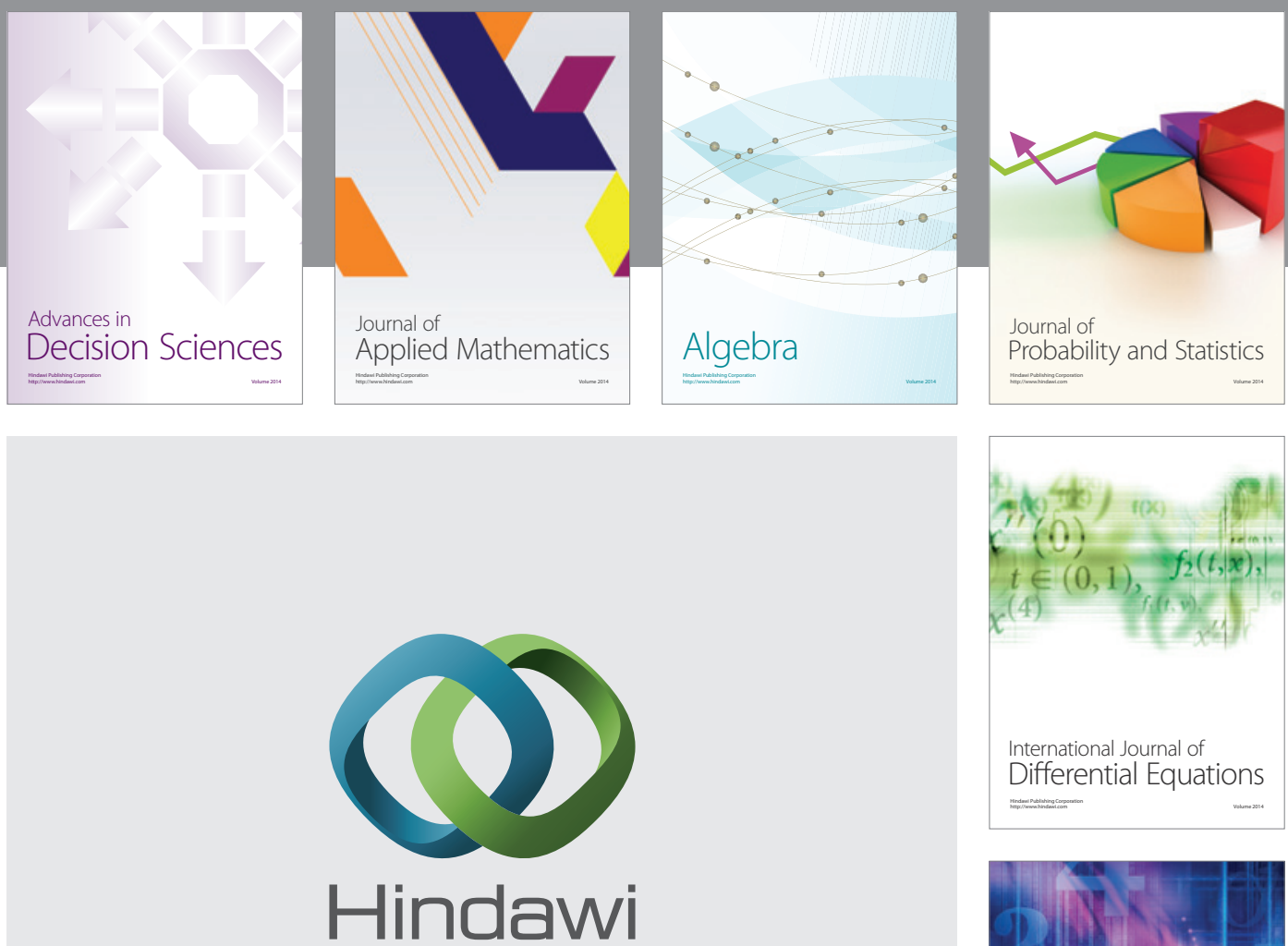

Submit your manuscripts at http://www.hindawi.com
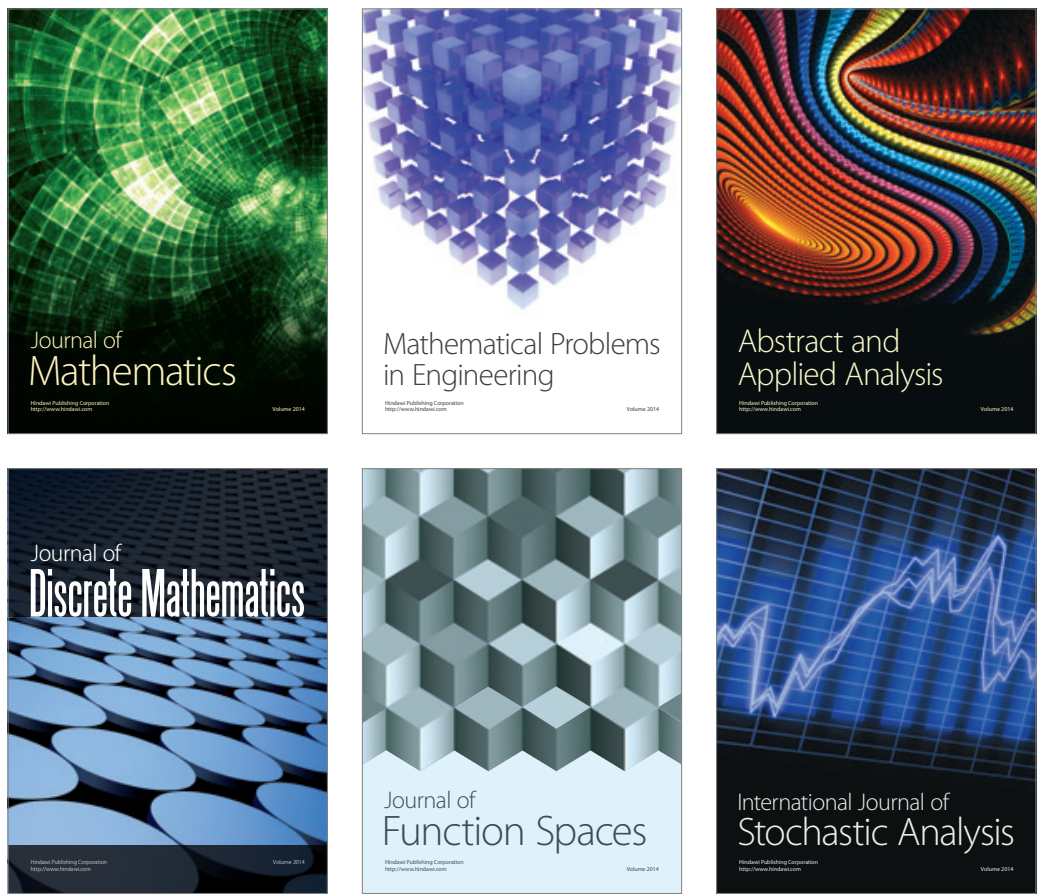

Journal of

Function Spaces

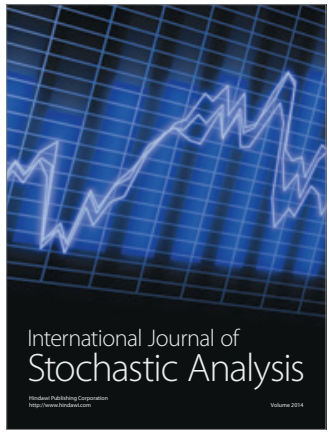

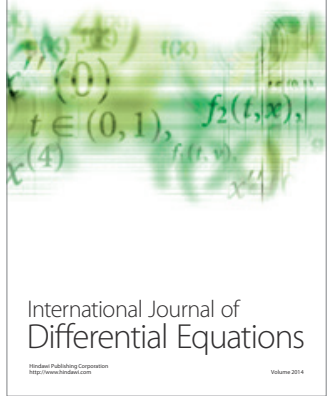
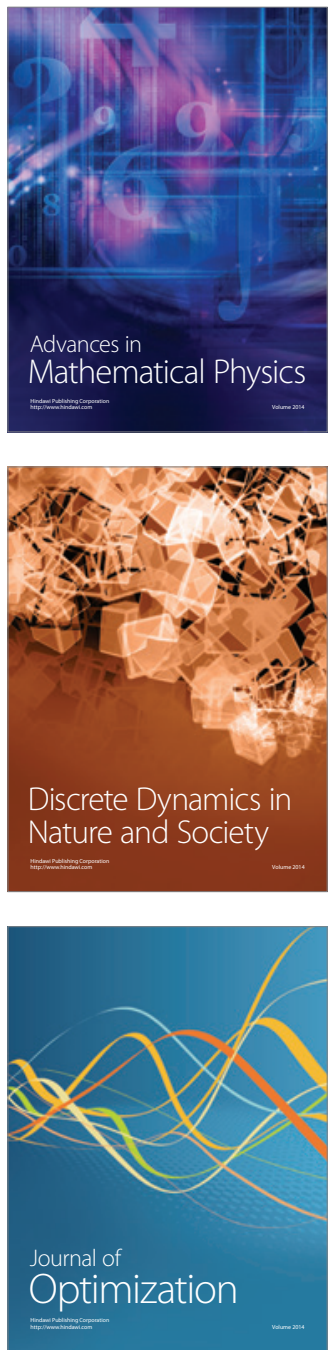